\section{Expression of SP-C and Ki67 in lungs of preterm infants dying from respiratory distress syndrome}

\author{
H. Lu, ${ }^{1,2}$ W. Li, ${ }^{2}$ G. Shao, ${ }^{3}$ H. Wang ${ }^{2}$ \\ 'Department of Pediatrics, the Affiliated \\ Hospital of Jiangsu University, Zhenjiang; \\ 2Department of Pediatrics, Tongji Medical \\ College, Huazhong University of Science \\ and Technology, Wuhan; \\ ${ }^{3}$ Department of Cell Biology, School of \\ Medicine, Jiangsu University, Zhenjiang, \\ China
}

\section{Abstract}

This study aimed at exploring the expression of Surfactant protein-C (SP-C) and Ki67 in autopsy lung tissues of premature infants dying from respiratory distress syndrome (RDS) who were exposed to mechanical ventilation and elevated oxygen concentrations. The possible influence of pulmonary surfactant (PS) on the expression of SP-C and Ki67 was also investigated. Thirty preterm infants were selected who were histologically and clinically diagnosed as RDS. Preterm infants with RDS were divided into 4 groups, according to the time of death: infants ventilated for 1-3 days, $4-8$ days, $9-16$ days and $>6$ days. Five premature infants died within 1 day after delivery for non- pulmonary reasons served as controls. The expression of SP-C and Ki67 in lungs was detected by immunohistochemistry. Compared with the control group, the expression of SP-C and Ki67 in RDS infants decreased significantly after 1-3 days of ventilation, but increased after 4 days and reached peak value after 9-16 days. No significant difference in the expression of SP-C and Ki67 was found between infants treated with PS and those without. Thus our results suggest SP-C and Ki67 may have participated in the pulmonary pathological process in ventilated/oxygen treated preterm infants with RDS, and exogenous surfactant had no effect on the expression of SP$\mathrm{C}$ and Ki67 in the lungs of ventilated/oxygen treated preterm infants with RDS.

\section{Introduction}

The neonatal respiratory distress syndrome (RDS) is one of the most common problems of premature infants. It is also a leading cause of mortality in infants and accounts for $20 \%$ of all neonatal deaths. ${ }^{1}$ Nowadays, mortality rates have dramatically decreased in infants with RDS due to the use of mechanical ventilation, supplemental oxygen and surfactant replacement therapy. ${ }^{2}$ However, prolonged mechanical ventilation and supplemental oxygen are both risk factors for the progression of RDS towards bronchopulmonary dysplasia (BPD), ${ }^{3}$ which is characterized by impaired alveolarization, abnormal vascularization and decreased lung function. ${ }^{4}$ Surfactant deficiency and immaturity are pivotal risk factors for developing RDS and subsequently BPD in preterm infants. ${ }^{5}$ Pulmonary surfactant (PS) is a mixture of lipids and proteins. There are four surfactant specific proteins (SPs), SP-A, SP-B, SP-C and SP-D. SP-C is generally deemed as the surfactant protein most specifically expressed in type-II alveolar epithelial cells and plays an important role in surfactant function. ${ }^{6}$ Ki67 is a nuclear antigen associated with cell proliferation and acts as a good proliferation marker of proliferating cells. ${ }^{7}$ In animal models, it has been demonstrated that SP-C and Ki67 may have significant changes in lungs exposed to hyperoxia. ${ }^{8,9}$ In post-mortem lung specimens of short-term ventilated preterm infants, the expression of Ki67 was threefold higher compared with control subjects. ${ }^{10}$ However, the influence of SP-C and Ki67 on lung tissue cells in preterm infants with RDS exposed to mechanical ventilation and elevated oxygen concentrations have yet to be determined. Recently, it has become increasingly obvious that exogenous surfactant preparations have significant effects on cell physiology, suppression of pro-inflammatory cytokines and regulation of the pulmonary host defense, ${ }^{11,12}$ but the influence of surfactant on the expression of SP-C and Ki67 is still unknown. The present study was conducted to examine the expression of SP-C and Ki67 in autopsy lung tissues of premature infants with RDS who were exposed to mechanical ventilation and elevated oxygen concentrations. The possible influence of surfactant on the expression of SP-C and Ki67 was also investigated.

\section{Materials and Methods}

\section{Subjects}

Autoposy lung specimens were selected in the files of the Department of Pathology in Tongji Medical College, Huazhong University of Science and Technology from the years 1995-2005. The study protocol was approved by the Ethics Committee of the Department of Tongji Hospital, Tongji Medical College, Huazhong University of Science and Technology, and written informed consent was obtained from at least one parent. Samples
Correspondence: Hong-yan Lu, Department of Pediatrics, the Affiliated Hospital of Jiangsu University, Zhenjiang 212001, China.

Tel. +86.15862979315.

E-mail: lhy5154@163.com.

Key words: respiratory distress syndrome, surfactant protein-C, Ki67, preterm.

Acknowledgement: this research was supported by Natural Science Foundation of Jiangsu Province, China (BK2011485), the Social Development Foundation of Zhenjiang, China (SH2011022), and National Nature Science foundation of China (81170573/H0417).

Contributions: HL, research design, manuscript writing, data acquisition and analysis, revising and final approval; WL, HW, research performing, data analysis; GS, critical manuscript revision, article drafting. All authors were involved in critically revising the manuscript for important intellectual content and approved the final version.

Received for publication: 24 January 2012

Accepted for publication: 24 April 2012

This work is licensed under a Creative Commons Attribution NonCommercial 3.0 License (CC BYNC 3.0).

(C) Copyright H. Lu et al., 2012

Licensee PAGEPress, Italy

European Journal of Histochemistry 2012; 56:e35 doi:10.4081/ejh.2012.e35

selected for analysis were infants with gestational ages less than 32 weeks and birth weight less than $1500 \mathrm{~g}$. The diagnosis of RDS was based on clinical and radiographic findings. Exclusion criteria were pulmonary malformation, pneumonia, chorioamnionitis, and inflammation of the umbilical cord or placentitis. Thirty cases fulfilled these criteria and had received conventional mechanical ventilation and supplemental oxygen $\left(\mathrm{FiO}_{2} 0.6-1.0\right)$ within $6 \mathrm{~h}$ after birth. All patients were still on mechanical ventilation and oxygen when they died. In order to investigate whether changes in the expression of SP-C and Ki67 depend on the duration of ventilation at elevated oxygen concentrations, preterm infants with RDS were divided into 4 groups, infants ventilated for 1-3 days, 4-8 days, 9-16 days and $>16$ days, respectively. Ventilator records were reviewed. The mean daily inspiratory oxygen fraction $\left(\mathrm{FiO}_{2}\right)$, mean airway pressure (MAP) and oxygenation index (OI) were determined and averaged over the duration of ventilator treatment. The oxygenation index was calculated as follows: oxygenation index $=[$ (MAP $x$ $\left.\left.\mathrm{FiO}_{2}\right) / \mathrm{PaO}_{2}\right] \times 100$. In addition, surfactant treatment (poractant alfa Curosurf, Chiesi Farmaceutici, Parma, Italy) and no surfactant 
treatment were considered in mechanically ventilated/oxygen-treated preterm infants with RDS. The preparation and composition of Curosurf have been previously described. ${ }^{13}$ Premature infants who died within $24 \mathrm{~h}$ of delivery for non-pulmonary reasons served as controls, which included 2 stillborn fetuses, 2 severe asphyxia, and 1 complicated congenital heart diseases. Clinical details of the infants are given in Table 1.

\section{Fixation, dehydration, embedding and stained with haematoxylin}

Autopsies were performed within 1 day after death. The lung samples were fixed in 10\% neutral buffered formalin, embedded in paraffin, and stored at room temperature. Paraffin embedded lung tissue was cut into $4 \mu \mathrm{m} \mathrm{sec-}$ tions and stained with haematoxylin.

\section{Immunohistochemistry}

Immunohistochemistry demonstration of SP-C and Ki67 were performed employing a rabbit polyclonal antibody against SP-C (Santa Cruz Biotechnology Inc., Santa Cruz, CA, USA) and a mouse monoclonal antibody against Ki67 (BD Biosciences, San Jose, CA, USA). Four $\mu \mathrm{m}$ sections were deparaffinized, rehydrated, and microwaved. The sections were then treated with $0.3 \%$ hydrogen peroxide in methanol for 30 min and blocked with either normal goat serum for 30 min. Primary antibody to SP-C (diluted 1:150 in PBS) or to Ki67 (diluted 1:100 in PBS) was added, and the sections were incubated overnight at $4^{\circ} \mathrm{C}$. Confirmation of specificity of the antibodies was performed by substituting the primary antibody with neutral isotonic PBS. The secondary antibody, biotinylated mouse/anti-rabbit IgG for SP-C, biotinylated goat/anti-mouse IgG for Ki67 was applied for $30 \mathrm{~min}$, followed by the avidin-biotin-peroxidase complex (Elite ABC Kit, Beijing Zhongshan Biotechnology Co., Beijing, China) for $30 \mathrm{~min}$, at room tem- perature following the manufacturer's instructions. The immunoreaction was visualized by incubating the sections for $4 \mathrm{~min}$ in a $0.1 \%$ 3,3 '- diaminobenzidine and $0.02 \%$ hydrogen peroxide solution. The sections were lightly counterstained with Mayer's haematoxylin and mounted. The cells with yellow brown particle deposition in cytoplasm (SP-C) or cell nucleus (Ki67) were judged to be positive. The analysis of SP-C and Ki67 expression was performed with HMIAS-2000 high-resolution color pathological imaging system. Five fields were randomly selected in each slide under a light microscope $(\times 200)$ for average absorption optical density (A value).

\section{Statistical analysis}

All data are presented as means $\pm \mathrm{SD}(\overline{\mathrm{x}} \pm \mathrm{s})$. The difference among groups was assessed by AVONA and Student's $t$-test. SPSS 12.0 package was used in the analysis. Differences were considered significant when a $\mathrm{P}$ value of $<0.05$ was reached.

\section{Results}

\section{Pulmonary pathology in premature infants with respiratory distress syndrome}

Amongst those infants with RDS, acute alveolar damage with alveolar collapse, epithelial necrosis, interstitial edema and hemorrhage, desquamation of pneumocytes and hyaline membrane formation was observed in infants who died at the ages of 1-3 days. Persistence of acute alveolar damage with unresolved hyaline membrane, interstitial and intra alveolar hemorrhage was observed in infants who died at the ages of 4-8 days. Histological evidence of less hyaline membrane, type-II alveolar epithelial cells hyperplasia, re-epithelialization was mainly in infants who died at the ages of 9-16 days. While decreased number of alveoli, increased variability in alveolar size, and increased interstitial thickness were the hallmark features of infants at the ages of $>16$ days. Among those infants with exogenous surfactant, no significant difference was identified in the pulmonary pathology in infants with RDS.

\section{Expression of SP-C and $\mathrm{Ki} 67$ in lungs of preterm infants with respiratory distress syndrome treated with ventilation at elevated oxygen concentrations}

In lung tissue sections from preterm infants with RDS who required mechanical ventilation and supplemental oxygen, the positive staining of SP-C was restricted to the type II alveolar epithelial cells (Figure $1 \mathrm{~A}-\mathrm{E}$ ), and Ki67 positive nuclei were preferentially localized in alveolar and bronchiolar epithelial cells and fibroblasts (Figure $2 \mathrm{~A}-\mathrm{E}$ ).

Compared with control group, the expression of SP-C and Ki67 in lungs from preterm infants with RDS, who were ventilated at elevated oxygen concentrations, decreased significantly after 1-3 days of ventilation, but increased after 4-8 days of ventilation, as well as after 9-16 days and $>16$ days of ventilation. It reached peak value after 9-16 days of ventilation. The expression of Ki67 in lungs from preterm infants with RDS, who were ventilated at elevated oxygen concentrations, had similar tendency with that of SP-C (Figure 3 A,B).

\section{Influence of surfactant on the expression of SP-C and $\mathrm{Ki} 67$}

The expression of SP-C as well as Ki67 in lungs from preterm infants with RDS who received surfactant was compared with those who did not. The expression of SP-C in infants who received surfactant was higher than that

Table 1. Clinical details of study population.

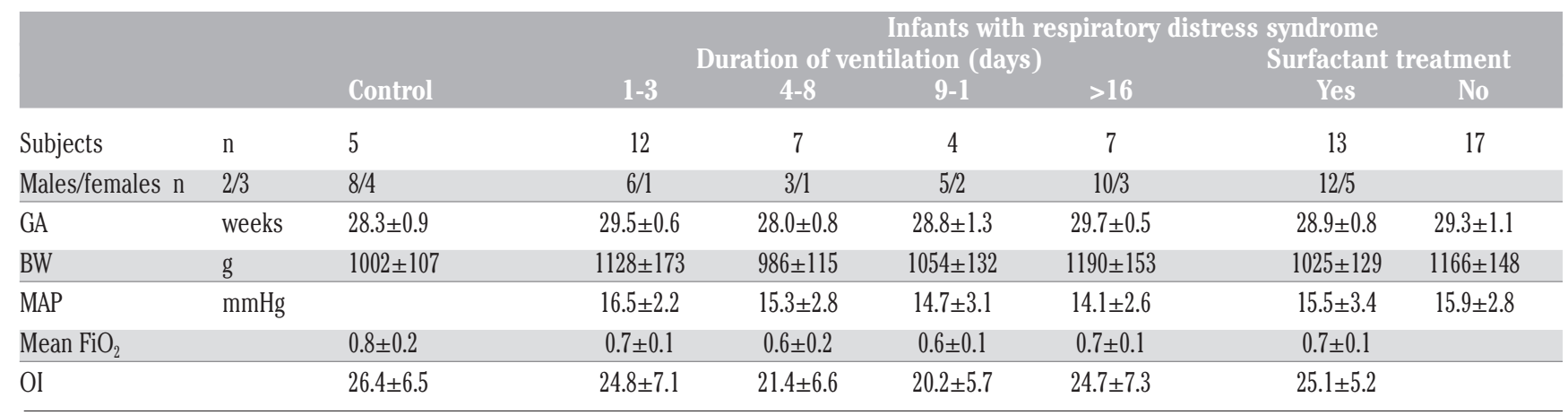

$\mathrm{GA}$, gestational age; $\mathrm{BW}$, birth weight; MAP, mean airway pressure; $\mathrm{FiO}_{2}$, inspiratory oxygen fraction; OI, oxygenation index. Data are expressed as mean $\pm \mathrm{SD}$. 
found in patients not treated with surfactant, but statistical analysis revealed no significance. Exogenous surfactant also had no effect on the expression of Ki67 (Figure 4).

\section{Discussion}

Despite the use of exogenous surfactant and the advances in neonatal intensive care, BPD remains a common chronic disease of very low birth weight preterm infants. Lung immaturity, oxidative stress, mechanical ventilation, and pulmonary inflammation are considered to be major factors involved in the pathogenesis of BPD. In our present study, the pulmonary pathology at different phases in premature infants with RDS who required mechanical ventilation and supplemental oxygen conforms to the main feature of different stages at development from RDS toward BPD as described by Rosan and Dik. ${ }^{14,15}$ The development from RDS to BPD is characterized by extensive tissue
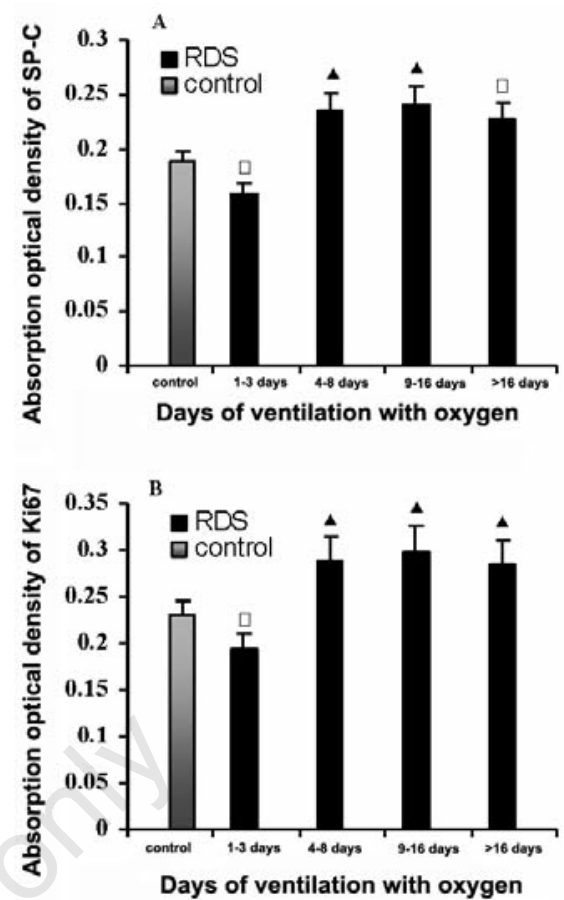

Figure 3. Column diagram showing the effect mechanical ventilation at elevated oxygen concentrations on SP-C (A), and Ki67 (B) in the lungs of preterm infants with respiratory distress syndrome. Compared with premature infants who died without lung disorders, SP-C and Ki67 decreased significantly after 1-3 days of ventilation, but increased significantly after 4-8 days as well as 9-16 days and $>16$ days. $\square \mathrm{P}<0.05, \Delta \mathrm{P}<0.01$ vs control.

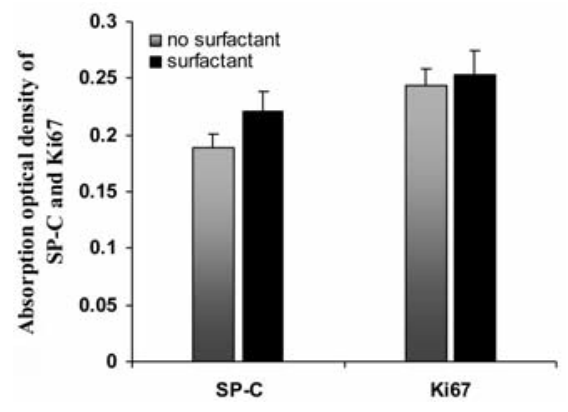

Figure 4. Column diagram showing the effect of exogenous surfactant on the expression of SP-C and $\mathrm{Ki} 67$ in the lungs of preterm infants with respiratory distress syndrome. No significantly difference was found between infants treated with surfactant and those without. drome after 4 days duration of mechanical ventilation at elevated oxygen concentrations than in premature infants who died without lung disorders.

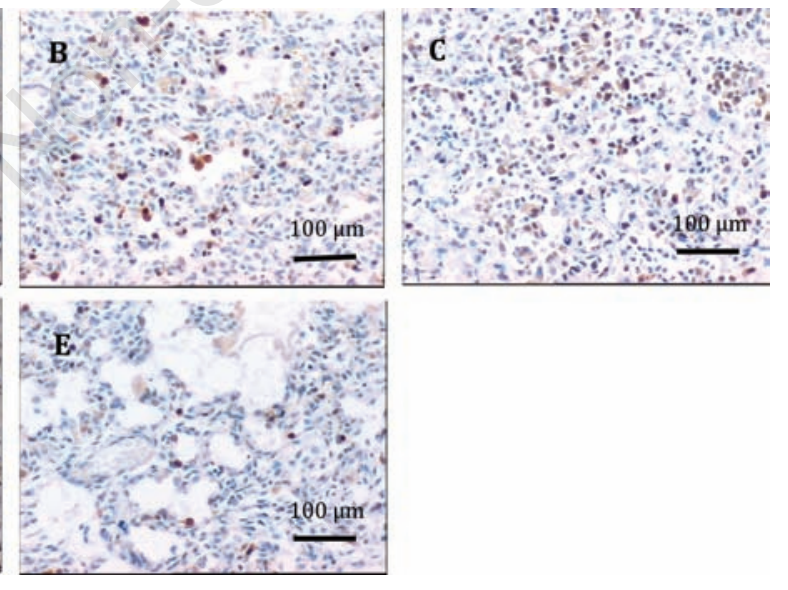
vated oxygen concentrations for 1-3 days, 4-8 days, 9-16 days and $>16$ days, respectively. E) From premature infants who died without lung disorders; the positive staining of Ki67 was preferentially localized in alveolar and bronchiolar epithelial cells and fibroblasts;

OPEN 
remodeling and can be divided into four phases based on days after birth: acute (2-4 d), regenerative (4-8 d), transitional (8-16 d), and chronic ( $>16 \mathrm{~d})$. A main feature of the acute and regenerative phase is persistence of acute alveolar damage with hyaline-membrane formation and hyaline membrane unresolved. The transitional phases are characterized by re-epithelialization of the denuded alveoli, alveoli primarily lined with type-II alveolar epithelial cells. During the chronic phase, hyaline membranes were virtually absent. The alveolus is covered exclusively with type-II-like alveolar epithelial cells; but the number of alveoli decreases and interstitial fibrosis is also a prominent feature. The lung findings in our present study manifested the main pulmonary pathology features of different stages of RDS leading to BPD, reaffirming the role of mechanical ventilation and supplemental oxygen as risk factors.

Normal lung development, which occurs as a series of complex tightly regulated events, can be divided into a number of stages. The saccular stage occurs between 26 and 36 weeks in uterus, begins with marked enlargement of the peripheral airways as the acinar tubules dilate and the walls thin, resulting in increased gas exchanging surface area. ${ }^{16}$ Lamellar bodies in type II cells increase and further maturation into type I cells occurs. At this time, the balance between proliferation and differentiation of lung cells plays an important role in normal cell turnover and lung development. Using a line of transgenic mice exposed to hyperoxia, Yee and co-workers found that perinatal hyperoxia adversely affects alveolar development by disrupting the proper timing of type II cell proliferation and differentiation into type I cells. ${ }^{17}$ This can explain why alveolar remodeling normally occurs in adults after hyperoxia whereas it is permanently disrupted in newborns; SP-C is synthesized by the type II alveolar epithelial cells. Mature SP-C is stored in the lamellar bodies and finally secreted into the alveolar space. SP-C plays a role in surface tension reduction, by promoting rapid insertion and spreading of lipids into the surface film. ${ }^{18}$ Exogenous surfactant preparations consisting of phospholipids and the hydrophobic proteins SP-B and SP-C are effectively used for the treatment of RDS of preterm infants. $.^{19} \mathrm{SP}-\mathrm{C}$ is generally recognized as the surfactant protein most specifically expressed in type II cells. As a result, measuring the levels of SP-C can be used as an estimation of the ability of the lung epithelial cells to produce surfactant as well as the identification for type II cells. ${ }^{20}$ In our experiment, compared with controls, the expression of SP-C and Ki67 in lungs from preterm infants with RDS, who were ventilated at elevated oxygen concentrations, decreased significantly after 1-3 days of ventilation.
There are at least two explanations accounting for this observed reduction in expression. One explanation is that the synthesis of pulmonary surfactant in premature infants with RDS is insufficient; the other explanation believes that this may be due to the accumulation of reactive oxygen species. Hyperoxia produces free radicals, causes cellular edema and cellular necrosis, and inhibits type II cells proliferation by down-regulating the expression of Ki67 at the early stage of exposure. The injury or decrease of the type II cells is likely to result in the declines in alveolar shortage of SP-C. The low levels of SP-C results in poor pulmonary compliance, atelectasis, decreased gas exchange, and severe hypoxia and acidosis. Premature infants with RDS must expend a great deal of effort to expand their lungs with each breath, and have higher requirements of $\mathrm{FiO}_{2}$ and MAP when they exposed to mechanical ventilation. The duration of mechanical ventilation/oxygen treatment plays a critical role in preterm infants. With increasing duration of mechanical ventilation at elevated oxygen levels, a considerably increasing expression of SP-C and Ki67 could be demonstrated nearly at the same time, and reach peak value after 9-16 days of ventilation. Consequently, the present study demonstrates that prolonged mechanical ventilation and supplemental oxygen may induce proliferation of the pulmonary cells, including type-II alveolar epithelial cells. Similarly, De Paepe et al. described higher expression of Ki67 in postmortem lung specimens of short-term and long-term ventilated preterm infants $;^{10}$ this is in good agreement with the findings in our present study. In rat lung, under prolonged hyperoxic conditions, increased lung cell proliferation in premature rats was also observed. ${ }^{21}$ White et al. ${ }^{22}$ also confirm our observation that hyperoxia increases the tissue expression of surfactant protein mRNAs in newborn rats, an increase in SP-C might be attributable to proliferation of alveolar type II cells.

Various studies in animal models and cell culture have emphasized the physiological and immunomodulatory effects of pulmonary surfactant. ${ }^{23}$ Results from clinic studies indicate that surfactant replacement therapy may result in a rapid improvement in dynamic compliance and oxygenation. It may also decrease duration of assisted ventilation and supplemental oxygen administration in the newborns with RDS, ${ }^{24}$ but has no effect on the incidence of BPD. ${ }^{25}$ The effect of exogenous surfactant on the expression of SP-C and Ki67 in ventilated/oxygen-treated lungs remains an interesting issue. Previously, it was shown that surfactant inhibited proliferation in fibroblast cultures..$^{26}$ Exogenous surfactants used to treat preterm infants with RDS can have profound effects on cell viability and DNA synthesis,
DNA synthesis was increased in A549 and rat type II cells. ${ }^{27}$ However, in the lungs of preterm infants with RDS exposed to mechanical ventilation and supplemental oxygen, exogenous surfactant has no effect on the pulmonary pathology, as well as the expression of SP-C and Ki67 in lung tissues. This result is in good agreement with what May and coworkers have found in premature infants with $\mathrm{RDS}^{28}$ Therefore, proliferation was obviously not influenced by surfactant treatment in the lungs of mechanically ventilated/oxygen-treated preterm infants with RDS. Exogenous surfactant cannot stop the progression of RDS toward BPD.

In summary, our results suggest that SP-C and Ki67 may have participated in the pulmonary pathological process in ventilated/oxygen treated preterm infants with RDS, and exogenous surfactant had no effect on the expression of SP-C and Ki67.

\section{References}

1. Sun B, Ma L, Liu X, Gao X, Ni L. Development of neonatal respiratory and intensive care: Chinese perspectives. Neonatology 2011;101:77-82.

2. Ma L, Liu C, Wang Y, Li S, Zhai S, Gu X, et al. Mortality of neonatal respiratory failure related to socioeconomic factors in Hebei province of China. Neonatology 2011; 100:14-22.

3. Collaborative study group for bronchopulmonary dysplasia of prematurity in China. Incidence and risk factors of bronchopulmonary dysplasia in premature infants in 10 hospitals in China. [Article in Chinese]. Zhonghua Er Ke Za Zhi 2011; 49:655-62.

4. Kramer BW, Lievense S, Been JV, Zimmermann LJ. From classic to new bronchopulmonary dysplasia. Ned Tijdschr Geneeskd 2010;154:1024.

5. Verder H, Bohlin K, Kamper J, Lindwall R, Jonsson B. Nasal CPAP and surfactant for treatment of respiratory distress syndrome and prevention of bronchopulmonary dysplasia. Acta Paediatr 2009;98:1400-8.

6. Orgeig S, Hiemstra PS, Veldhuizen EJ, Casals C, Clark HW, Haczku A, et al. Recent advances in alveolar biology: evolution and function of alveolar proteins. Respir Physiol Neurobiol 2010;173:S43-54.

7. Hitchman E, Hodgkinson C, Roberts D, Ashton G, Yunus Z, Byers R, et al. Effect of prolonged formalin fixation on immunohistochemical staining for the proliferation marker Ki67. Histopathology 2011; 59:1261-3. 
8. Lu HY, Shao GB, Li WB, Wang H. Effects of hyperoxia on transdifferentiation of primary cultured type II alveolar epithelial cells from premature rats. In vitro Cell Dev Biol Anim 2011;47:64-72.

9. Lu HY, Chang LW, Wang H, Li WB, Wang H. Effects of hyperoxia on the proliferation and cell cycle of type II alveolar epithelial cells from premature rat. [Article in Chinese]. Chin Pediatr Emerg Med 2007; 14:32-4.

10. De Paepe ME, Mao Q, Powell J, Rubin SE, DeKoninck P, Appel N, et al. Growth of pulmonary microvasculature in ventilated preterm infants. Am J Respir Crit Care Med 2006;173:204-11.

11. Golubev AM, Perepelitsa SA, Smerdova EF, Moroz VV. Lung morphological changes in premature neonates with hyaline membrane disease due to the use of exogenous surfactants during mechanical ventilation. [Article in Russian]. Arkh Patol 2010; 72:34-8.

12. Donn SM, Dalton J. Surfactant replacement therapy in the neonate: beyond respiratory distress. Respir Care 2009; 54:1203-8.

13. Verder H, Robertson B, Greisen G, Ebbesen F, Albertsen P, Lundstrøm K, et al. Surfactant therapy and nasal continuous positive airway pressure for newborns with respiratory distress syndrome. Danish-Swedish Multicenter Study Group. N Engl J Med 1994;331:1051-5.

14. Rosan RC. Hyaline membrane disease and a related spectrum of neonatal pneumopathies. Perspect Pediatr Pathol 1975;2: 15-60.
15. Dik WA, De Krijger RR, Bonekamp L, Naber BA, Zimmermann LJ, Versnel MA. Localization and potential role of matrix metalloproteinase-1 and tissue inhibitors of metalloproteinase- 1 and -2 in different phases of bronchopulmonary dysplasia. Pediatr Res 2001;50:761-6.

16. Kotecha S. Lung growth: implications for the newborn infant. Arch Dis Child Fetal Neonatal 2000;82:69-74.

17. Yee M, Vitiello PF, Roper JM, Staversky RJ, Wright TW, McGrath-Morrow SA, et al. Type II epithelial cells are critical target for hyperoxia-mediated impairment of postnatal lung development. Am J Physiol Lung Cell Mol Physiol 2006;291:1101-11.

18. Na Nakorn P, Meyer MC, Flach CR, Mendelsohn R, Galla HJ. Surfactant protein $\mathrm{C}$ and lung function: new insights into the role of alpha-helical length and palmitoylation. Eur Biophys J 2007;36:477-89.

19. van Kaam AH, De Jaegere AP, Borensztajn D, Rimensberger PC, Neovent Study Group. Surfactant replacement therapy in preterm infants: a European survey. Neonatology 2011;100:71-7.

20. Glasser SW, Eszterhas SK, Detmer EA, Maxfield MD, Korfhagen TR. The murine SP-C promoter directs type II cell-specific expression in transgenic mice. Am J Physiol Lung Cell Mol Physiol 2005;288: 625-32.

21. Gao Y, Xue XD, Li JY, Wang N. Expression and roles of CDK4 and p21 in lung tissues of premature rats with hyperoxia-induced chronic lung disease. [Article in Chinese]. Zhongguo Dang Dai Er Ke Za Zhi 2007; 9:595-600.
22. White CW, Greene KE, Allen CB, Shannon JM. Elevated expression of surfactant proteins in newborn rats during adaptation to hyperoxia. Am J Respir Cell Mol Biol 2001;25:51-9.

23. Chroneos ZC, Sever-Chroneos Z, Shepherd VL. Pulmonary surfactant: an immunological perspective. Cell Physiol Biochem 2010;25:13-26.

24. Stevens TP, Harrington EW, Blennow M, Soll RF. Early surfactant administration with brief ventilation vs. selective surfactant and continued mechanical ventilation for preterm infants with or at risk for respiratory distress syndrome. Cochrane Database Syst Rev 2007;17:CD003063.

25. Husain AN, Siddiqui NH, Stocker JT. Pathology of arrested acinar development in postsurfactant bronchopulmonary dysplasia. Hum Pathol 1998;29:710-7.

26. Thomassen MJ, Antal JM, Barna BP, Divis LT, Meeker DP, Wiedemann HP. Surfactant downregulates synthesis of DNA and inflammatory mediators in normal human lung fibroblasts. Am J Physiol 1996; 270:159-63.

27. Wemhöner A, Jennings P, Haller T, Rüdiger M, Simbruner G. Effect of exogenous surfactants on viability and DNA synthesis in A549, immortalized mouse type II and isolated rat alveolar type II cells. BMC Pulm Med 2011;11:11.

28. May M, Ströbel P, Preisshofen T, Seidenspinner S, Marx A, Speer CP. Apoptosis and proliferation in lungs of ventilated and oxygen-treated preterm infants. Eur Respir J 2004;23:113-21. 